\title{
Stereoacuity in children with anisometropic amblyopia
}

\section{Citation}

Wallace, David K., Elizabeth L. Lazar, Michele Melia, Eileen E. Birch, Jonathan M. Holmes, Kristine B. Hopkins, Raymond T. Kraker, Smith, L.E., et al. 2011. Stereoacuity in Children with Anisometropic Amblyopia. Journal of American Association for Pediatric Ophthalmology and Strabismus 15, no. 5: 455-461. doi:10.1016/j.jaapos.2011.06.007.

\section{Published Version}

doi:10.1016/j.jaapos.2011.06.007

\section{Permanent link}

http://nrs.harvard.edu/urn-3:HUL.InstRepos:33776200

\section{Terms of Use}

This article was downloaded from Harvard University's DASH repository, and is made available under the terms and conditions applicable to Other Posted Material, as set forth at http:// nrs.harvard.edu/urn-3:HUL.InstRepos:dash.current.terms-of-use\#LAA

\section{Share Your Story}

The Harvard community has made this article openly available.

Please share how this access benefits you. Submit a story.

\section{Accessibility}




\title{
Stereoacuity in children with anisometropic amblyopia
}

\author{
David K. Wallace, MD, MPH ${ }^{a}$, Elizabeth L. Lazar, MS, MPH ${ }^{b}$, Michele Melia, ScM ${ }^{b}$, Eileen E. \\ Birch, PhD ${ }^{\mathrm{C}}$, Jonathan M. Holmes, BM, BCh ${ }^{\mathrm{d}}$, Kristine B. Hopkins, OD, MSPH ${ }^{\mathrm{e}}$, Raymond T. \\ Kraker, MSPH $^{\mathrm{b}}$, Marjean T. Kulp, OD, MS ${ }^{\dagger}$, Yi Pang, PhD, ODg, Michael X. Repka, MD, MBA ${ }^{\mathrm{h}}$, \\ Susanna M. Tamkins, ODi, and Katherine K. Weise, OD, MBA ${ }^{e,{ }^{*}}$ on behalf of the Pediatric \\ Eye Disease Investigator Group
}

aDuke University Eye Center, Durham, North Carolina bJaeb Center for Health Research, Tampa, Florida 'Retina Foundation of the Southwest, Dallas, Texas 'Mayo Clinic College of Medicine, Rochester, Minnesota eUniversity of Alabama at Birmingham School of Optometry, Birmingham,

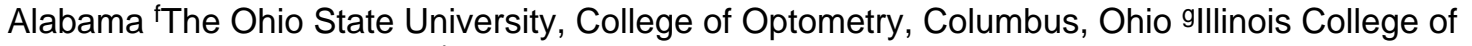
Optometry, Chicago, Illinois hJohns Hopkins University School of Medicine, Baltimore, Maryland 'Bascom Palmer Eye Institute, Miami, Florida

\section{Abstract}

Purpose-To determine factors associated with pretreatment and posttreatment stereoacuity in subjects with moderate anisometropic amblyopia.

\begin{abstract}
Methods-Data for subjects enrolled in seven studies conducted by the Pediatric Eye Disease Investigator Group were pooled. The sample included 633 subjects aged 3 to $<18$ years with anisometropic amblyopia, no heterotropia observed by cover test, and baseline amblyopic eye acuity of 20/100 or better. A subset included 248 subjects who were treated with patching or Bangerter filters and had baseline stereoacuity testing and outcome examinations. Multivariate regression models identified factors associated with baseline stereoacuity and with outcome stereoacuity as measured by the Randot Preschool Stereoacuity test.
\end{abstract}

Results-Better baseline stereoacuity was associated with better baseline amblyopic eye acuity $(P<0.001)$, less anisometropia $(P=0.03)$, and anisometropia due to astigmatism alone $(P<$ $0.001)$. Better outcome stereoacuity was associated with better baseline stereoacuity $(P<0.001)$ and better amblyopic eye acuity at outcome $(P<0.001)$. Among 48 subjects whose amblyopic eye visual acuity at outcome was $20 / 25$ or better and within one line of the fellow eye, stereoacuity was worse than that of children with normal vision of the same age.

Conclusions-In children with anisometropic amblyopia of 20/40 to 20/100 inclusive, better posttreatment stereoacuity is associated with better baseline stereoacuity and better posttreatment amblyopic eye acuity. Even if their visual acuity deficit resolves, many children with anisometropic amblyopia have stereoacuity worse than that of nonamblyopic children of the same age.

() 2011 American Association for Pediatric Ophthalmology and Strabismus. Published by Mosby, Inc. All rights reserved. Correspondence: David K. Wallace, MD, MPH, c/o Jaeb Center for Health Research, 15310 Amberly Drive, Suite 350, Tampa, Florida 33647(pedig@jaeb.org).

A complete listing of the Pediatric Eye Disease Investigator Group is provided in e-Supplement 1, available at jaapos.org.

Publisher's Disclaimer: This is a PDF file of an unedited manuscript that has been accepted for publication. As a service to our customers we are providing this early version of the manuscript. The manuscript will undergo copyediting, typesetting, and review of the resulting proof before it is published in its final citable form. Please note that during the production process errors may be discovered which could affect the content, and all legal disclaimers that apply to the journal pertain.

The authors have no financial or conflicting interests in the subject of this report to disclose. 
One of the potential benefits of amblyopia treatment is improved stereoacuity, the discrimination of differences in depth based on binocular retinal disparity. It has been reported that baseline stereoacuity in amblyopic patients is associated with anisometropia type and magnitude, ${ }^{1}$ anisometropia magnitude alone, ${ }^{1,2}$ and treatment-related improvement in amblyopic eye acuity. ${ }^{3}$ One study found that improvement in stereoacuity is associated with improvement in amblyopic eye visual acuity. ${ }^{4}$

The Pediatric Eye Disease Investigator Group (PEDIG) has enrolled more than 3,000 amblyopic patients into treatment trials, prospectively collecting visual acuity and stereoacuity data using standardized methods. These data provide an opportunity to determine what factors from among those measured are independently associated with pretreatment and posttreatment stereoacuity and to describe outcome stereoacuity in subjects whose amblyopic eye visual acuity after treatment was $20 / 25$ or better and within one line of the fellow eye visual acuity. This information may help clinicians identify children with the greatest potential for improvement in stereoacuity and may provide insight into the pathophysiology of amblyopia and subnormal binocular vision.

\section{Methods}

\section{Cohort Eligibility and Protocol Selection}

Data were pooled from seven randomized multicenter clinical trials of subjects ages 3 to $<18$ years in which Randot stereoacuity data were collected at baseline (Table 1$),{ }^{5-11}$ details of which are available on the PEDIG website (www.pedig.net). The studies were supported through a cooperative agreement with the National Eye Institute of the National Institutes of Health EY011751 and EY018810. The protocol and HIPAA-compliant informed consent forms were approved by institutional review boards, a parent or guardian of each study subject gave written informed consent, and subjects gave assent as required. The studies adhered to the tenets of the Declaration of Helsinki and are listed on www.clinicaltrials.gov under identifiers NCT00094692, NCT00094614, NCT00091923, NCT00315198, NCT00315302, NCT00315328, and NCT00525174. Inclusion in the baseline stereoacuity analysis was limited to subjects with moderate amblyopia (20/40 to 20/100 inclusive on the same day of baseline stereoacuity measurement) from anisometropia alone without a history or presence at enrollment of measurable heterotropia at distance or near fixation, resulting in a sample size of 633 subjects. For the purposes of inclusion in the treatment trials, anisometropia was defined as $\geq 0.50 \mathrm{D}$ of spherical equivalent difference or $\geq 1.50 \mathrm{D}$ difference in astigmatism in any meridian. Subjects with amblyopic eye visual acuity worse than 20/100 were excluded since they were expected to have little or no stereoacuity (as do normal subjects with induced monocular blur) ${ }^{12}$; those with manifest strabismus (evident by simultaneous prism and cover test at either distance or near) were also excluded since strabismus may also limit potential stereoacuity.

The cohort for the analyses of outcome stereoacuity consisted of 248 subjects ages 3 to $<13$ years from 3 of the 7 protocols $8,10,11$ that included a stereoacuity measurement at both baseline and at the primary outcome examination (Table 1). The outcome cohort had the same inclusion criteria as the baseline cohort, with the additional requirements of a measurable Randot Preschool Stereoacuity score at outcome and treatment with either patching or Bangerter filters during the clinical trial. Subjects treated with atropine were excluded from the outcome stereoacuity analysis because residual blur from atropine at the outcome examination may have led to underestimation of true stereoacuity in some cases. 


\section{Measurement of Anisometropia, Visual Acuity, and Stereoacuity}

All measurements were performed with the subjects wearing appropriate refractive corrections, which consisted of spectacle wear for all but one study that permitted contact lens use at enrollment. The refractive corrections were based on a cycloplegic refraction that was performed within 6 months of study enrollment. Hypermetropia could not be undercorrected by more than $+1.50 \mathrm{D}$ spherical equivalent, and the reduction in plus had to be symmetrical in the two eyes.

In five of the seven protocols, baseline acuity measurements were taken following a 16week period with appropriate refractive correction or after visual acuity in the amblyopic eye was stable (defined as two consecutive visual acuity measurements by the same testing method at least 4 weeks apart with no improvement of one line or more). In the remaining two protocols, there was no requirement for stability of visual acuity with spectacle wear prior to additional treatment.

For the analyses, the type of anisometropia was defined as belonging to one of three groups. The first group, "Spherical equivalent anisometropia alone," was defined as $\geq 0.50 \mathrm{D}$ interocular difference in spherical equivalent in the absence of astigmatic anisometropia $\geq 0.75 \mathrm{D}$ interocular difference in either Jackson-cross cylinder powers of $\mathrm{J}_{0}$ (with-the-rule or against-the-rule) or $\mathrm{J}_{45}$ (oblique). ${ }^{13,14}$ The second group, "Astigmatic anisometropia alone," was defined as $\geq 0.75 \mathrm{D}$ interocular difference in either $\mathrm{J}_{0}$ or $\mathrm{J}_{45}$ in the absence of $\geq 1.50 \mathrm{D}$ interocular difference in spherical equivalent. In this category, some subjects had spherical equivalent anisometropia $\geq 0.50 \mathrm{D}$ and $<1.50 \mathrm{D}$, and we assumed their amblyopia was primarily on the basis of astigmatic anisometropia. The third group, "Combined anisometropia," was defined as $\geq 1.50 \mathrm{D}$ interocular difference in spherical equivalent and $\geq 0.75 \mathrm{D}$ interocular difference in either $\mathrm{J}_{0}$ or $\mathrm{J}_{45}$. The amount of anisometropia was also expressed as vector dioptric difference (VDD), resulting in a single number representing the difference in refraction between the two eyes. ${ }^{15}$

Visual acuity was measured using either the Amblyopia Treatment Study HOTV (ATSHOTV $)^{16,17}$ protocol for subjects aged 3 to $<7$ years or the Electronic Early Treatment for Diabetic Retinopathy Study (E-ETDRS) ${ }^{18}$ protocol for older subjects. Visual acuity at the outcome examination was measured using the same testing method performed at baseline regardless of age at follow-up. All visual acuity scores were expressed as $\operatorname{logMAR}$ for the analyses.

Baseline and outcome stereoacuity were measured using the Randot Preschool Stereoacuity ${ }^{19}$ test in all protocols (Stereo Optical Co. Inc, Chicago, IL). Subjects were given a pretest by presenting the black-and-white test plate and were asked to "point to the duck." Those unable to pass the pretest were excluded from this analysis. Subjects passing the pretest were next presented with shapes subtending 800 " stereopsis and were asked to name or match one of the random dot shapes that the tester pointed to. A black and white matching card was provided to allow the child to point at the matching shape. The tester pointed to a second random dot shape, and the child was asked to name or match that shape. A third shape was presented if needed to classify as a pass or fail at that level. Two correct answers (of a possible 3) were required to pass each level and proceed to the next stereoacuity level. Stereoacuity was recorded as the finest stereoacuity level at which 2 correct figures were identified (40", 60", 100", 200", 400", or 800"). If the subject was unable to pass the 800 " level, then stereoacuity was recorded as "nil."

\section{Statistical Methods}

For all analyses, stereoacuity scores in seconds of arc were converted to log values as follows: 40" (1.60), 60" (1.78), 100" (2.00), 200" (2.30), 400" (2.60), 800" (2.90); subjects 
with no detectable (nil) stereoacuity were assigned a value of 1600" (3.20). Logged stereoacuity scores were treated as a continuous measure for the primary analyses because, unlike rank-based analyses, the log scale retains information about the nonuniform scale intervals. Analyses were repeated using extreme values for nil (1000" and 10,000") and excluding subjects with nil stereoacuity at baseline to check the sensitivity of results to the value assigned to nil stereoacuity.

Covariates of age, baseline amblyopic eye acuity, anisometropia amount, and anisometropia type were selected for analysis based on biologic plausibility of an association with baseline stereoacuity. For the outcome stereoacuity analyses, factors of interest included age, baseline amblyopic eye acuity, anisometropia amount, anisometropia type, baseline stereoacuity, and amblyopic eye acuity at outcome. In addition, age was also treated as an adjustment covariate in both analyses, and hence included in all models regardless of statistical significance.

Baseline amblyopic eye acuity and baseline stereoacuity were similarly included as adjustment covariates in the outcome analyses. Although the outcome analyses evaluated level of stereoacuity at the outcome examination, the adjustment for baseline stereoacuity yields covariate estimates that are identical to those from the model evaluating change in stereoacuity at outcome adjusted for baseline stereoacuity. ${ }^{20}$

Multivariate linear regression models were fit to determine predictors of baseline and outcome stereoacuity, adjusting for potential confounders and including protocol as a fixed effect. ${ }^{21}$ Protocol also served as an adjustment for spectacle stability at enrollment, which was required in all but two of the studies included in the baseline analysis. Linearity assumptions were assessed for each continuous factor included in the multivariate regression models, adjusting for the other covariates. For the baseline stereoacuity analysis, there was a nonlinear relationship between age and baseline stereoacuity, so age was treated as a categorical covariate ( 3 to $<5,5$ to $<7,7$ to $<10$, and 10 to $<18$ years).

All possible two-way interaction terms of covariates were evaluated in the models and retained if meeting a statistical significance criterion of $P<0.001$, chosen to control for the large number of terms tested. Initial models included all factors of interest with nonsignificant factors removed from subsequent models using a backward stepwise elimination procedure. The final models included statistically significant factors of interest, as well as all adjustment covariates (age and protocol for the baseline analysis; age, protocol, and baseline amblyopic eye visual acuity for the outcome analysis). Adjusted mean stereoacuity was computed for all levels of the categorical predictors and statistically significant differences were identified using the Tukey-Kramer multiple-comparison procedure. $^{22}$ The 10 to $<18$ age group was not compared with the 3 to $<5$ or the 5 to $<7$ age groups since there was no overlap in the protocols across these age ranges. The interaction between protocol and each of the covariates was tested in the final model to check for homogeneity among protocols.

An analysis of covariance was used to compare the mean log stereoacuity at outcome between subjects with and without outcome amblyopic eye acuity of 20/25 or better and within one line of the fellow eye acuity (the better of fellow eye acuities at enrollment and outcome), adjusting for baseline stereoacuity, age, and protocol. Exact Wilcoxon rank-sum tests were used to compare ranked log stereoacuity scores in this subset of subjects with successful amblyopia treatment to those of normal children, stratified by age group. Normal data were based on findings from another study of 4,355 subjects, aged 3 to 18 years old, with permission from the authors. ${ }^{23}$ 
All baseline and outcome stereoacuity analyses were performed using SAS Version 9.1 (SAS Institute, Cary, NC).

\section{Results}

\section{Baseline Stereoacuity}

The characteristics of subjects included in the baseline stereoacuity analyses are listed in eSupplement 2 (available at jaapos.org). The mean baseline stereoacuity was $2.7 \mathrm{log}$ arcsec (between $400^{\prime \prime}$ and $800^{\prime \prime}$ ) for the overall cohort and $2.3 \mathrm{log}$ arc seconds (approximately $\left.200^{\prime \prime}\right)$ for those with measurable baseline stereoacuity $(\mathrm{N}=397) ; 236$ of 633 subjects (37\%) had no measurable baseline stereoacuity. In a multivariate model, better baseline stereoacuity was associated with the 5 to $<10$ age groups ( 5 to $<7$ vs 3 to $<5$ years, $P=0.01$; 7 to $<10$ vs 10 to $<18$ years, $P=0.04)$, better baseline amblyopic eye acuity $(P<0.001$, Figure 1), less anisometropia $(P=0.03)$ and anisometropia due to astigmatism alone $(P<$ 0.001 ) (e-Supplement 3, available at jaapos.org). Sensitivity analyses using extreme values for nil yielded similar results. There were no statistically significant interactions between any of the covariates in the baseline stereoacuity model ( $P \geq 0.14$ for all interactions).

Subjects with astigmatic anisometropia alone had better baseline stereoacuity (median 200") compared with those with just spherical equivalent anisometropia (median 800", $P<0.001$ ). Baseline stereoacuity was similar between the spherical equivalent anisometropia, and the combined spherical and astigmatic anisometropia (median 400") groups. The estimated effects of the predictors on baseline stereoacuity were consistent among protocols (data not shown).

\section{Outcome Stereoacuity after Amblyopic Treatment}

The characteristics of the 248 subjects included in the cohort for the analyses of outcome stereoacuity are given in e-Supplement 4 (available at jaapos.org). None of the subjects in this cohort had heterotropia observed at baseline, and 10 subjects had heterotropia observed at outcome (6 constant heterotropia, 4 intermittent). The mean stereoacuity at outcome after treatment was $2.4 \log$ arc seconds (between 200" and 400") with a mean baseline stereoacuity of $2.6 \mathrm{log} \operatorname{arcsec}\left(400^{\prime \prime}\right)$ and mean improvement of $0.2 \mathrm{log}$ arc seconds (approximately 0.7 of a level). Seventy subjects (28\%) improved at least 2 levels of stereoacuity (approximating the amount of change required to exceed test-retest variability), ${ }^{24}$ and 19 subjects (8\%) worsened at least 2 levels. Of 248 subjects, 154 (62\%) achieved at least 200" of stereoacuity, and 63 (25\%) achieved at least 60 " of stereoacuity.

In a multivariate model, better outcome stereoacuity was associated with better baseline stereoacuity $(P<0.001)$ and better amblyopic eye acuity at outcome $(P<0.001$, Figure 2$)$, adjusting for baseline amblyopic eye acuity $(P=0.66)$, age $(P=0.05)$ and protocol (eSupplement 5, available at jaapos.org). These factors remained significant predictors of outcome stereoacuity when subjects with no measurable stereoacuity at baseline were excluded from the analysis (results not shown), and sensitivity analyses using extreme values for nil (1000" and 10,000") yielded similar results. The estimated effects of the predictors on outcome stereoacuity were consistent among protocols.

Anisometropia type $(P=0.07)$ and magnitude of anisometropia $(P=0.71)$ were not statistically significant predictors of outcome stereoacuity. There were no statistically significant interactions between any of the covariates in the outcome stereoacuity model $(P$ $\geq 0.02$ for all interaction terms) based on our prespecified criteria. 


\section{Stereoacuity after Successful Amblyopia Treatment}

Of 48 subjects who achieved 20/25 or better amblyopic eye acuity that was within one line of the fellow eye visual acuity, 26 (54\%) had a Randot Preschool Stereoacuity score of 60 arcsec or better. Mean stereoacuity at outcome was better among these subjects compared to those who did not meet these outcome visual acuity criteria (2.1 log arcsec or between 100" and $200^{\prime \prime}$ vs $2.4 \log$ arcsec or between $200^{\prime \prime}$ and $400^{\prime \prime}, P<0.001$ ), adjusting for baseline stereoacuity, age and protocol. Figure 3 shows that age-specific stereoacuity of our successfully treated amblyopic subjects was worse than that of children with normal vision without amblyopia.

\section{Discussion}

In this study, better baseline stereoacuity was associated with better baseline amblyopic eye visual acuity, less anisometropia, and anisometropia due to astigmatism alone among subjects with anisometropic amblyopia and no heterotropia observed by cover test. Better outcome stereoacuity was associated with better baseline stereoacuity and better outcome amblyopic eye acuity.

We also observed some differences in baseline stereoacuity among age groups. Subjects aged 3 to $<5$ years had poorer baseline stereoacuity compared to that of subjects aged 5 to $<10$ years, and subjects aged 10 to $<18$ had poorer baseline stereoacuity compared to subjects aged 7 to $<10$ years after adjustment for potential confounders. The relatively poor stereoacuity of the youngest group might be explained by undetected microtropia or poorer testing performance. Despite the trends observed in e-Supplement 3, baseline stereoacuity among subjects aged 7 to $<10$ years was not statistically different from that of the 3 to $<5$ or 5 to $<7$ age groups. These comparisons were inadequately powered to detect an age effect and were dependent on a single protocol that enrolled subjects in all three age groups. Subjects ages 10 years and older might have had worse baseline stereoacuity because of undetected microtropia with identity, or such children who present at this age may more commonly have a resistant type of amblyopia, particularly if they had not improved with prior amblyopia treatment.

Factors predictive of outcome stereoacuity were not identical to those predictive of baseline stereoacuity. One important reason for this disparity is that the outcome stereoacuity model included an adjustment for baseline stereoacuity. As a result, any factor found to be associated with outcome stereoacuity in this model is by definition associated with change in stereoacuity with treatment.

The relationship between stereoacuity and amblyopic eye acuity may be because better visual acuity allows for better discrimination of stereoacuity test targets. Odell and colleagues ${ }^{12}$ found that stereoacuity was progressively degraded by increasing levels of induced monocular blur in 15 normal adults, and that random dot stereo test performance is particularly sensitive to degradation by monocular blur.

Other investigators have examined the relationship between stereoacuity and amblyopic eye visual acuity. Caputo and colleagues ${ }^{3}$ found that better baseline stereoacuity is predictive of improvement in amblyopic eye acuity, and they hypothesized that the presence of binocular vision at the first evaluation was a good prognostic indicator for visual recovery with optical correction alone. Lee and colleagues ${ }^{4}$ reported a significant linear relationship between stereoacuity improvement measured by the Titmus test and visual acuity improvement in patients with and without small angle or intermittent strabismus. 
Better baseline stereoacuity was associated with less anisometropia when expressed as vector dioptric difference. These data agree with findings of other investigators who found that baseline stereoacuity in amblyopic patients is associated with magnitude of anisometropia. ${ }^{1,2}$ Weakley reported that higher amounts of spherical anisometropia and higher amounts of cylindrical anisometropia were each associated with decreased stereoacuity in spectacle correction. ${ }^{1,2}$ Rutstein and Corliss ${ }^{2}$ found that binocularity of spectacle-corrected hyperopic patients decreased as the degree of anisometropia increased. ${ }^{1}$ One explanation for these findings is that anisometropia and aniseikonia (image size disparity) are obstacles to sensory fusion and development of stereoacuity. Lubkin and colleagues ${ }^{25}$ showed that anisometropia is associated with amblyopia, and the combination of anisometropia and aniseikonia is strongly associated with amblyopia. Jimenez and colleagues $^{26}$ and Oguchi and colleagues ${ }^{27}$ reported an association between induced aniseikonia and reduced stereoacuity. Dobson and colleages ${ }^{15}$ found that small interocular refractive error differences disrupt stereoacuity, whereas larger interocular refractive error differences are necessary to produce differences in interocular, best-corrected recognition acuity. They hypothesized that development of stereoacuity is particularly dependent on similarity in refractive error between fellow eyes, even in the absence of unilateral amblyopia. ${ }^{15}$

Among subjects whose amblyopic eye acuity at outcome was 20/25 or better and within one line of the fellow eye visual acuity, stereoacuity was worse than that of children with normal vision of the same age. These data support the premise that anisometropic amblyopia is associated with subnormal binocular development. Although visual acuity in the amblyopic eye can improve with treatment to normal or near-normal levels, a significant deficit in binocular function, as implied by reduced stereoacuity, persists in many children. This deficit in stereoacuity, despite normal visual acuity, might result from a central insult induced by anisometropia early in life, possibly manifesting as ongoing partial foveal suppression or a central limitation of binocular potential, and/or from a combination of other unknown factors; however, this analysis does not address whether stereoacuity would improve further with complete resolution of amblyopia and/or longer treatment duration. It is also possible that stereoacuity was limited because of undetected microtropia in an unknown number of children in our cohort.

It is noteworthy that only 48 of our 248 subjects with anisometropic amblyopia and no heterotropia observed on cover testing (19\%) demonstrated marked improvement of visual acuity to $20 / 25$ or better in the amblyopic eye. In contrast, Agervi and colleagues ${ }^{28}$ reported that 62 of 66 children (94\%) with anisometropic amblyopia achieved resolution of amblyopia (interocular difference of one line or less) after one year of treatment with spectacles alone or with spectacles in addition to Bangerter filter. They may have observed a higher rate of amblyopia resolution because their subjects were younger, had less severe amblyopia, and had longer treatment duration than our subjects. The protocols included in our analyses were not designed to produce the maximal possible improvement with treatment.

In conclusion, better baseline stereoacuity is associated with better baseline amblyopic eye visual acuity, less anisometropia, and anisometropia due to astigmatism alone. Better stereoacuity after amblyopia treatment is associated with better baseline stereoacuity and better amblyopic eye acuity at outcome. For many children with anisometropic amblyopia, subnormal stereoacuity persists after a course of treatment, even when their visual acuity deficit effectively resolves. 


\section{Supplementary Material}

Refer to Web version on PubMed Central for supplementary material.

\section{Acknowledgments}

Supported by the National Eye Institute of the National Institutes of Health, Department of Health and Human Services EY011751 and EY018810. The sponsor or funding organization had no role in the design or conduct of this research. The studies are listed on www.clinicaltrials.gov, under identifiers NCT00094692, NCT00094614, NCT00091923, NCT00315198, NCT00315302, NCT00315328, and NCT00525174.

\section{References}

1. Weakley DR Jr. The association between nonstrabismic anisometropia, amblyopia, and subnormal binocularity. Ophthalmology. 2001; 108:163-171. [PubMed: 11150283]

2. Rutstein RP, Corliss D. Relationship between anisometropia, amblyopia, and binocularity. Optom Vis Sci. 1999; 76:229-233. [PubMed: 10333185]

3. Caputo R, Frosini R, De Libero C, Campa L, Magro EF, Secci J. Factors influencing severity of and recovery from anisometropic amblyopia. Strabismus. 2007; 15:209-214. [PubMed: 18058358]

4. Lee SY, Isenberg SJ. The relationship between stereopsis and visual acuity after occlusion therapy for amblyopia. Ophthalmology. 2003; 110:2088-2092. [PubMed: 14597513]

5. Scheiman MM, Hertle RW, Beck RW, et al. Pediatric Eye Disease Investigator Group. Randomized trial of treatment of amblyopia in children aged 7 to 17 years. Arch Ophthalmol. 2005; 123:437447. [PubMed: 15824215]

6. Repka MX, Cotter SA, Beck RW, et al. Pediatric Eye Disease Investigator Group. A randomized trial of atropine regimens for treatment of moderate amblyopia in children. Ophthalmology. 2004; 111:2076-2085. [PubMed: 15522375]

7. Wallace DK, Edwards AR, Cotter SA, et al. Pediatric Eye Disease Investigator Group. A randomized trial to evaluate 2 hours of daily patching for strabismic and anisometropic amblyopia in children. Ophthalmology. 2006; 113:904-912. [PubMed: 16751033]

8. Pediatric Eye Disease Investigator Group. A randomized trial of near versus distance activities while patching for amblyopia in children aged 3 to less than 7 years. Ophthalmology. 2008; 115:20712078. [PubMed: 18789533]

9. Pediatric Eye Disease Investigator Group. Pharmacological plus optical penalization treatment for amblyopia: Results of a randomized trial. Arch Ophthalmol. 2009; 127:22-30. [PubMed: 19139333]

10. Scheiman MM, Hertle RW, Kraker RT, et al. Pediatric Eye Disease Investigator Group. Patching vs atropine to treat amblyopia in children aged 7 to 12 years: A randomized trial. Arch Ophthalmol. 2008; 126:1634-1642. [PubMed: 19064841]

11. Rutstein RP, Quinn GE, Lazar EL, et al. Pediatric Eye Disease Investigator Group. A randomized trial comparing Bangerter Filters and patching for the treatment of moderate amblyopia in children. Ophthalmology. 2010; 117:998-1004. [PubMed: 20163869]

12. Odell NV, Hatt SR, Leske DA, Adams WE, Holmes JM. The effect of induced monocular blur on measures of stereoacuity. J AAPOS. 2009; 13:136-141. [PubMed: 19071047]

13. Harvey EM, Miller JM, Dobson V, Tyszko R, Davis AL. Measurement of refractive error in Native American preschoolers: Validity and reproducibility of autorefraction. Optom Vis Sci. 2000; 77:140-149. [PubMed: 10772231]

14. Thibos LN, Wheeler W, Horner D. Power vectors: An application of fourier analysis to the description and statistical analysis of refractive error. Optom Vis Sci. 1997; 74:367-375. [PubMed: 9255814]

15. Dobson V, Miller JM, Clifford-Donaldson CE, Harvey EM. Associations between anisometropia, amblyopia, and reduced stereoacuity in a school-aged population with a high prevalence of astigmatism. Invest Ophthalmol Vis Sci. 2008; 49:4427-4436. [PubMed: 18539935]

16. Holmes JM, Beck RW, Repka MX, et al. The amblyopia treatment study visual acuity testing protocol. Arch Ophthalmol. 2001; 119:1345-1353. [PubMed: 11545641] 
17. Moke PS, Turpin AH, Beck RW, et al. Computerized method of visual acuity testing: Adaptation of the amblyopia treatment study visual acuity testing protocol. Am J Ophthalmol. 2001; 132:903909. [PubMed: 11730656]

18. Beck RW, Moke PS, Turpin AH, et al. A computerized method of visual acuity testing: Adaptation of the early treatment of diabetic retinopathy study testing protocol. Am J Ophthalmol. 2003; 135:194-205. [PubMed: 12566024]

19. Birch E, Williams C, et al. the ALSPAC "Children in Focus" Study Team. Random dot stereoacuity of preschool children. J Pediatr Ophthalmol Strabismus. 1997; 34:217-222. [PubMed: 9253735]

20. Laird N. Further comparative analyses of pretest-posttest research designs. Am Stat. 1983; 37:329_ 330.

21. Verbeke, G.; Molenberghs, G. Estimation of the marginal model. In: Linear Mixed Models for Longitudinal Data. New York, MY: Springer-Verlag; 2000. p. 41-54.

22. Kramer CY. Extension of multiple range tests to group means with unequal numbers of replications. Biometrics. 1956; 12:307-310.

23. Birch E, Williams C, Drover J, et al. Randot Preschool Stereoacuity test: normative data and validity. J AAPOS. 2008; 12:23-26. [PubMed: 17720573]

24. Adams WE, Leske DA, Hatt SR, Holmes JM. Defining real change in measures of stereoacuity. Ophthalmology. 2009; 116:281-285. [PubMed: 19091410]

25. Lubkin V, Kramer P, Meininger D, Shippman S, Bennett G, Visintainer P. Aniseikonia in relation to strabismus, anisometropia, and amblyopia. Binocul Vis Strabismus Q. 1999; 14:203-207. [PubMed: 10553113]

26. Jimenez JR, Ponce A, del Barco LJ, Diaz JA, Perez-Ocon F. Impact of induced aniseikonia on stereopsis with random-dot stereogram. Optom Vis Sci. 2002; 79:121-125. [PubMed: 11871399]

27. Oguchi Y, Mashima Y. The influence of aniseikonia on the VEP by random-dot stereogram. Acta Ophthalmol (Copenh). 1989; 67:127-130. [PubMed: 2728863]

28. Agervi P, Kugelberg U, Kugelberg M, Simonsson G, Fornander M, Zetterstrom C. Treatment of anisometropic amblyopia with spectacles or in combination with translucent Bangerter filters. Ophthalmology. 2009; 116:1475-1480. [PubMed: 19500854] 


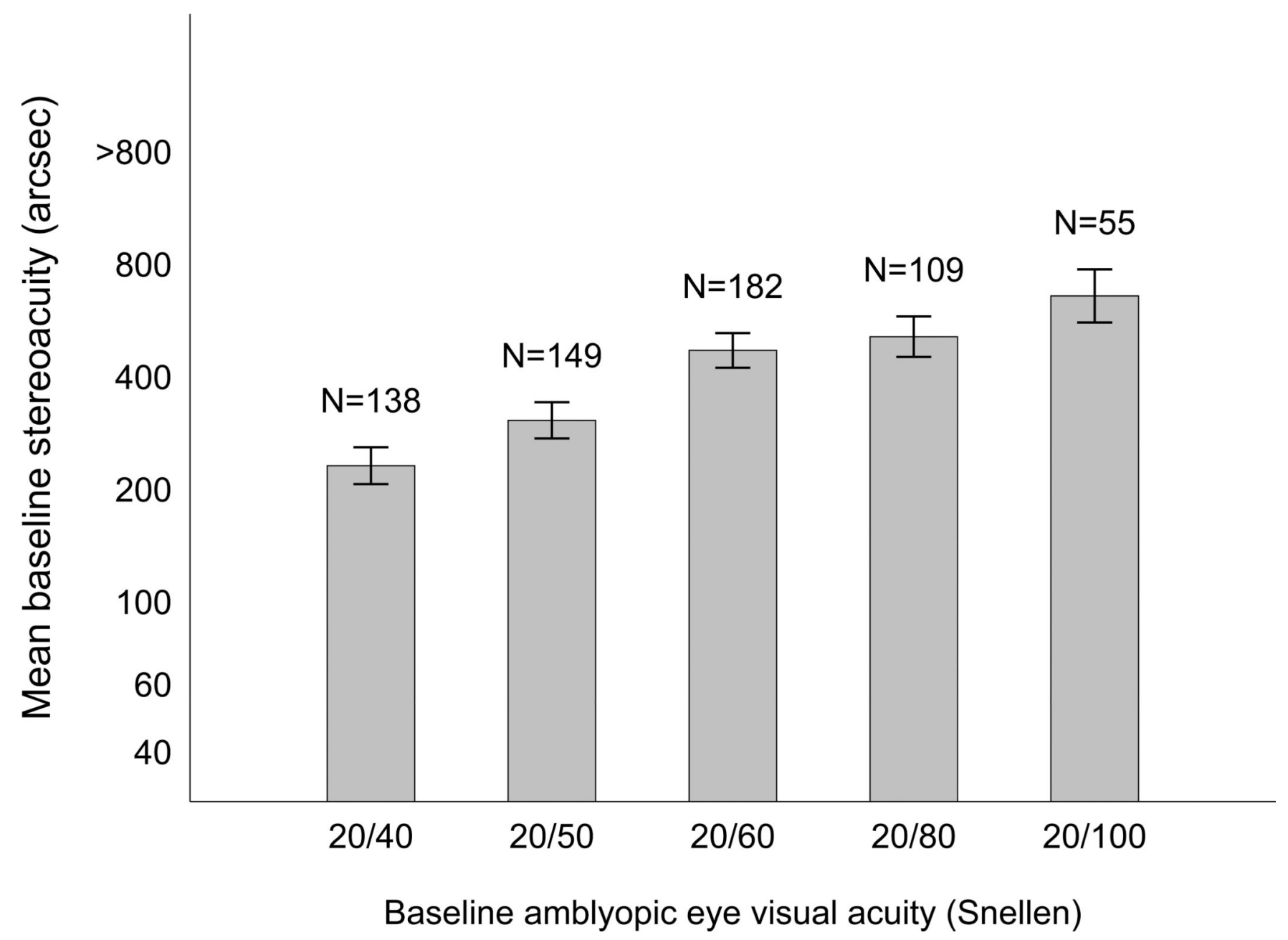

FIG 1.

Relationship between baseline amblyopic eye visual acuity and mean baseline stereoacuity, adjusting for the effect of other covariates. Mean baseline stereoacuity (log arcsec) was computed for each level of baseline visual acuity, adjusting for age group, magnitude of anisometropia, anisometropia type, and protocol. Better stereoacuity was associated with better amblyopic eye visual acuity $(P<0.001)$. 


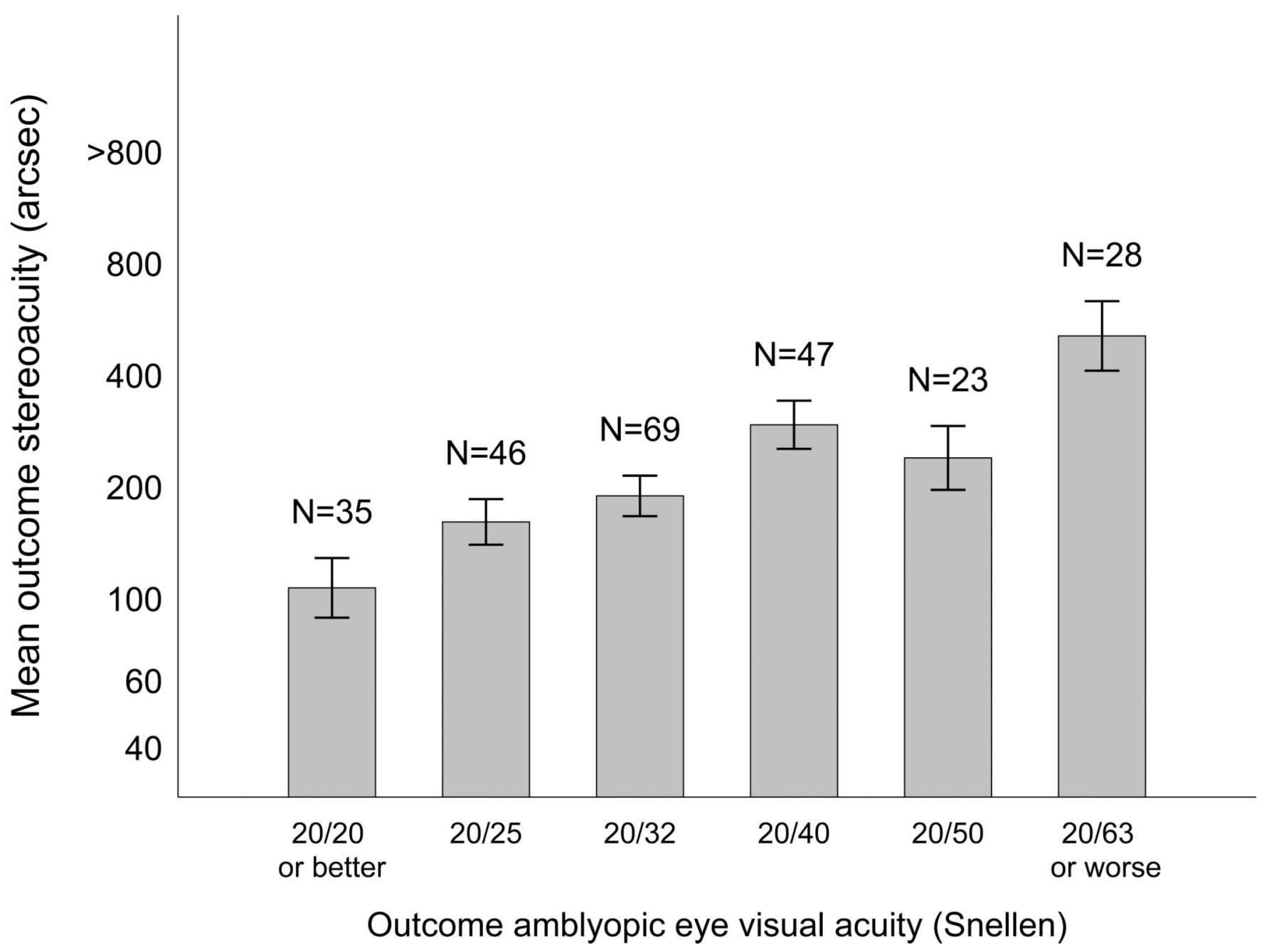

FIG 2.

Relationship between outcome amblyopic eye visual acuity and mean outcome stereoacuity after treatment, adjusting for the effect of other covariates. Mean stereoacuity at outcome after treatment (log arcsec) was computed for each level of amblyopic acuity eye visual at outcome, adjusting for age, baseline amblyopic eye acuity, baseline stereoacuity, and protocol. Better outcome stereoacuity was associated with better amblyopic eye visual acuity at outcome with treatment $(P<0.001)$. 


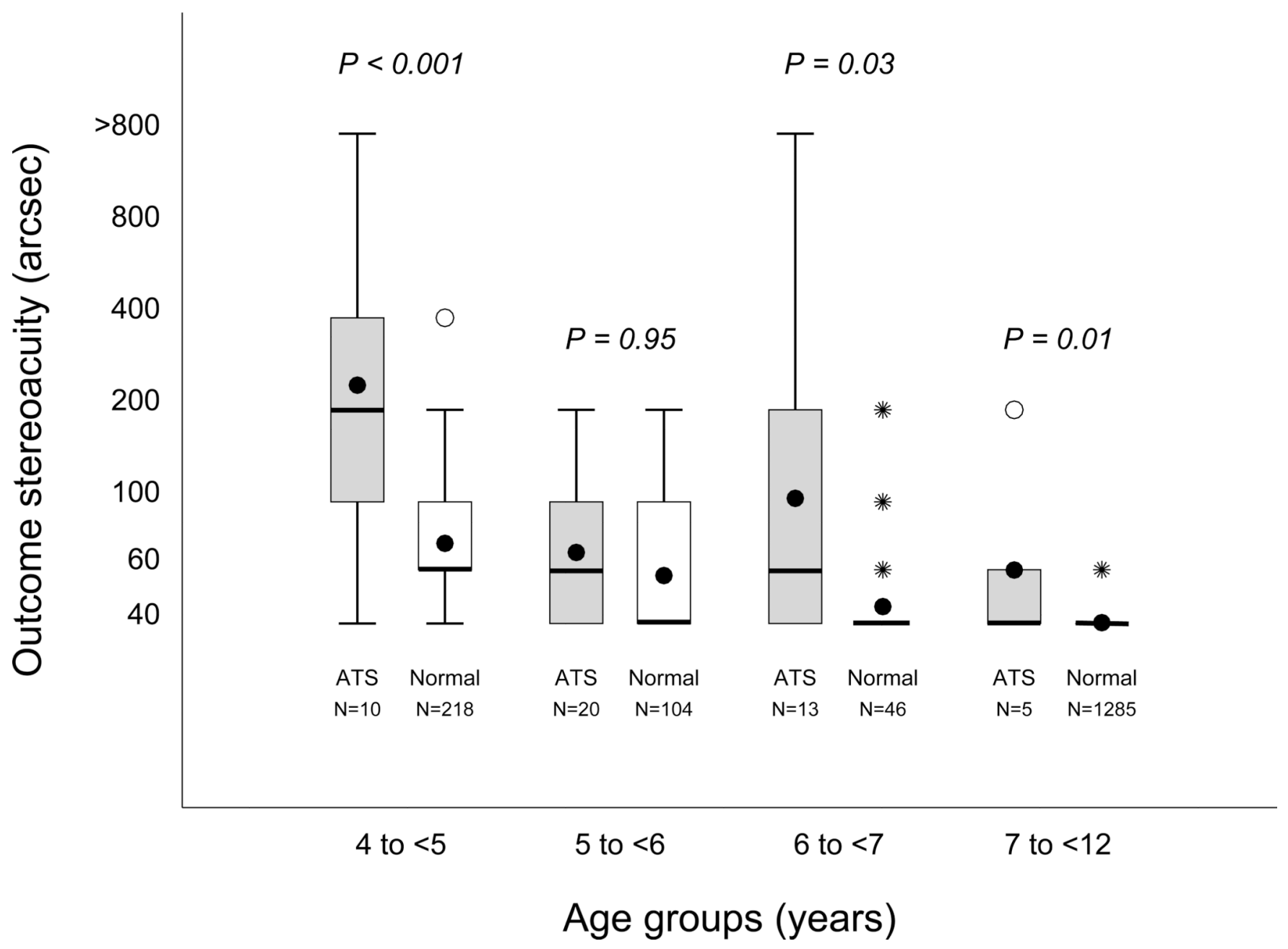

FIG 3.

Age group comparison of stereoacuity among subjects with amblyopic eye visual acuity of $20 / 25$ or better and within one line of the fellow eye visual acuity compared to normal children. For each age group, the box on the left (gray) is the distribution of stereoacuity scores for the Amblyopia Treatment Study subjects and that on the right (white) represents the normal children. The top and bottom of each box represent the 25th and 75th percentiles of the data, the group medians are represented by a bolded line, and the group means are represented by a dot. For some of the box plots, the median also corresponds to the 25th and/ or 75th percentile. The bars extending above and below each box represent 1.5 times the interquartile range (difference between the 25th and 75th percentiles). The open circles represent near statistical outliers, and the asterisks indicate far outliers. $P$ values are from the age-matched comparison of stereoacuity among children with amblyopic eye visual acuity of 20/25 or better and within one line of the fellow eye visual acuity at outcome compared to that of normal children based on the Wilcoxon exact rank-sum test. 


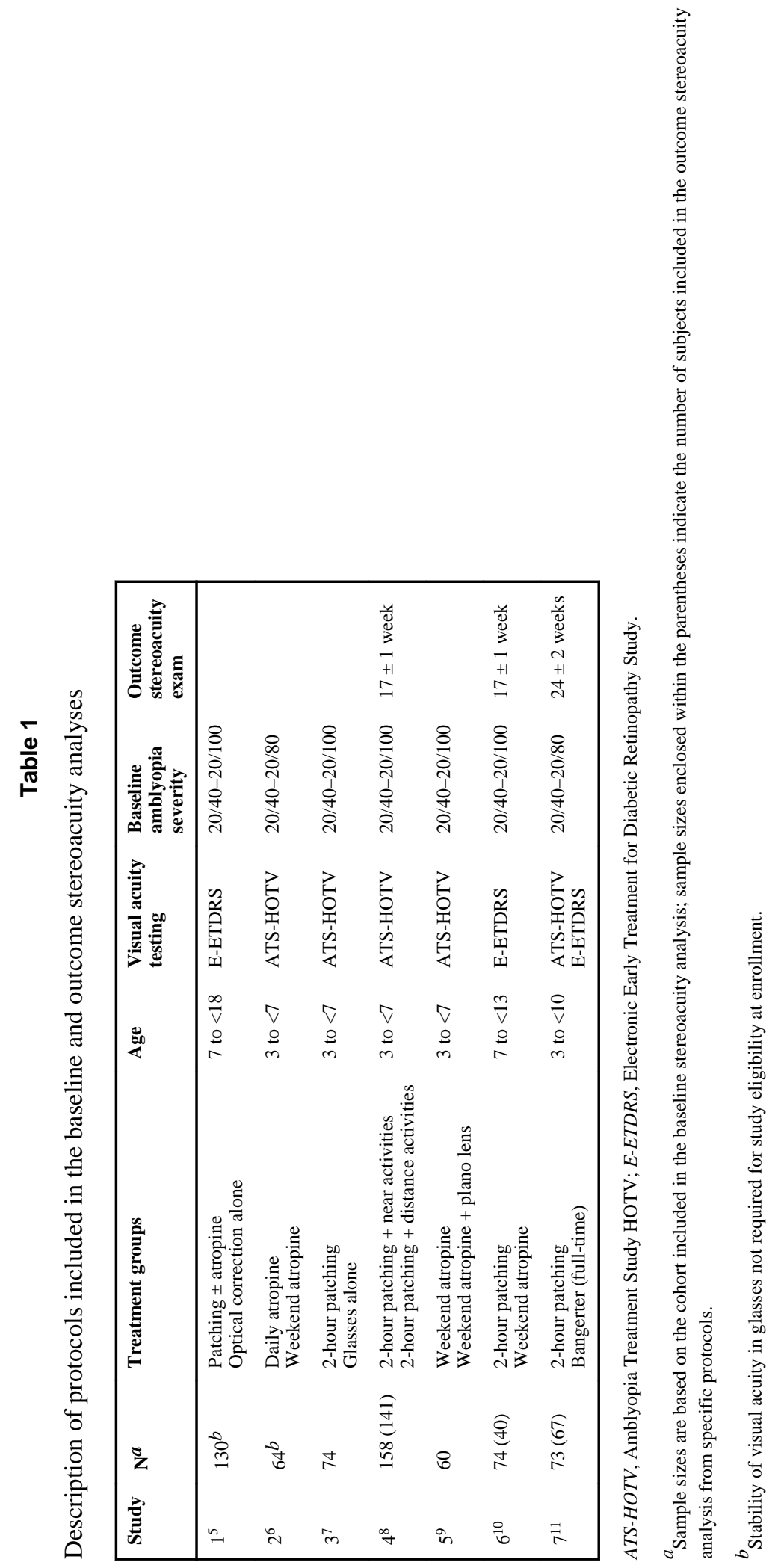

J AAPOS. Author manuscript; available in PMC 2012 October 1. 